\title{
Intelligent Ambulance Fleet Management System
}

\author{
${ }^{1}$ Monica Chhabria, ${ }^{2}$ Latika Wadhwa, ${ }^{3}$ Shruti Dhumale, ${ }^{4}$ Omkar Patinge, \\ ${ }^{5}$ Gresha Bhatia \\ ${ }^{1,2,3,4,4}$ Student Computer Engineering, Vivekanand Education Society Institute of Technology, \\ Mumbai, India \\ ${ }^{5,}$ Deputy HOD, Computer Engineering Vivekanand Education Society Institute of Technology, \\ Mumbai, India
}

\begin{abstract}
The time after a medical mishap if used optimally serves as a measure of the effectiveness of any ambulance service provider system. For a minimal loss of life recovery actions should be taken in time. In spite of advancement of technology in today's world, the service providers are not well equipped. Also, problems like routing problems and traffic congestions hamper their speedy recovery action in real time. Our aim is to save maximum number of lives in an emergency by reducing the reaction time by various means. Pre-registration and extracting the real-time location helps to reduce the reaction time. Another approach is by providing the shortest path to the ambulance driver. We identified that the current traffic situation is the biggest challenge for the ambulance to reach in time. Thus coordination with the traffic control room is essential in solving this problem. The proposed system is fully automated and thus allows to book an ambulance, to which system allocates ambulance and inform TCR in case of extreme emergency.
\end{abstract}

Keywords: Ambulance; GPS; shortest path; placement

\section{INTRODUCTION}

Human asset is dependably of gigantic support for developing nation like India. Despite the fact that our nation is soaked with 121 Crore populace, relatively few are paying heed to the more secure existence of a safety system. Transportation of a patient to clinic in crisis appears to be very basic yet in genuine it is truly troublesome amid pinnacle hours. A critical operation for the treatment of crisis episodes is the steering of reacting vehicles to occurrence locales and afterward to the nearest hospitals. GIS innovation can bolster crisis responders to give productive reaction in less time through illuminating the guiding problems. The effective administration of ambulances with a specific end goal to accomplish quick transportation of patients to the suitable hospital is an imperative part of the nature of well being administrations offered to natives.

Finishing a powerful directing and districting of ambulances will minimize their reaction time. Transporting the patient to the hospital is an essential undertaking since research demonstrates that there is a cozy relationship between the emergency vehicle reaction time and patient mortality. The more drawn out the time, the higher the mortality. In a thickly populated region, streets amid surge hours are regularly congested, rescue vehicle in participation process are all the time stuck in a road turned parking lot, in this way deferring the season of conveying.

The proposed system allows the user to book an ambulance in minimum time frame with few clicks. The system will allocate the nearest ambulance by checking the constraints set by the user. The nearest hospital will be allocated to the ambulance considering real time traffic data in mind. The shortest path will be provided to the ambulance driver by the system. In case of extreme emergency, the traffic control room will be notified about the path details to clear the traffic as much as possible. The system strives to improve the system efficiency, with a particular focus on reducing the response time.

\section{LITERATURE SURVEY}

There are a number of projects that focus on solving similar problems. Dah-Ming Shiah and Shu-Wen Chen [1] combined both deterministic and capacities to propose a model for placement of the 
ambulance. They proposed a model which setups ambulance site based on population density by deciding a preset distance to be covered and a preset population to be served. The algorithm proposed is an extension of local set covering models. The disadvantages of the model are that it requires detailed spatial information which is difficult to get. Another disadvantage is the border effect because of the insufficient data sets obtained from neighboring countries.

In next research [2], a prototype is proposed that detects the accident spot using GSM and GPS technology. The ambulance is provided with bio sensors to provide a patient's condition to the nearest hospital which uses Zigbee. The nearest hospital is found out using Dijkstra's Shortest Path Algorithm in NS2 Simulator. Once the nearest hospital is selected the traffic signals on the shortest path are put to ON state using RF receivers at the signals and RF receivers in the Ambulance. The disadvantage of this model is that there can be delays due to sending messages through GSM.

In another work by Yisong Yue and Lavanya Marla and Ramayya Krishnan [3], a strategy for ambulance allocation and dynamic redeployment is proposed. Thus, by processing the emergency requests the utility of an allocation is measured. This approach leads to exponentially huge action space. Despite this complexity, using the Greedy allocation algorithm leads to better results. Further, it is proposed that myopic redeployment algorithm is more effective as the randomness in the future depends purely on exogenous factors.

\section{Challenges of Existing System}

\subsection{Operator Centered}

In the current system the operators respond to the requests through the phone. This system takes a lot of time and human efforts to gather all the necessary information regarding the patient and location details. Even though the emergency cases have increased, increasing the number of operators is not profitable and feasible.

\subsection{Location Accuracy}

Many a times the location provided by the caller to the operator is not accurate, which may result in increased delay. The caller may try to provide a location using any popular landmark that the operator may not know. Also, if the ambulance driver thus not know the location or the shortest path with real time traffic to reach the given location or hospital.

\subsection{Managing Large Group of Callers}

In an operator centered scenario, when the number of callers increase at a particular time, it becomes difficult for the operators to respond to all of them. In such scenarios the efficiency of the system degrades due to the decrease in the number of customers served.

\subsection{Report Generation and Analysis}

As the system is paper based, interactive weekly or monthly report generation will require lots of human efforts and time. Analyzing the reports and efficiency is difficult. Also, we cannot backup the data and hence in case of disaster the data will be lost.

\section{Proposed Work}

The proposed structure of the system comprises of five basic modules, namely, Registration, Ambulance Booking, Request Processing, Placement and Analysis. The Ambulance booking module allows only the registered users to book an ambulance. The Request processing module takes the request details of the ambulance booking module and focuses on allocating the ambulance and providing the shortest path to the ambulance driver while coordinating with the TCR in case of traffic. Also the request processing module takes into consideration the ambulance placement strategies (for cardiac and accidents). The placement strategy considers the number of registered users, population density and accident prone areas for placing the ambulance so that the system can provide maximum benefits and reduce response time. 


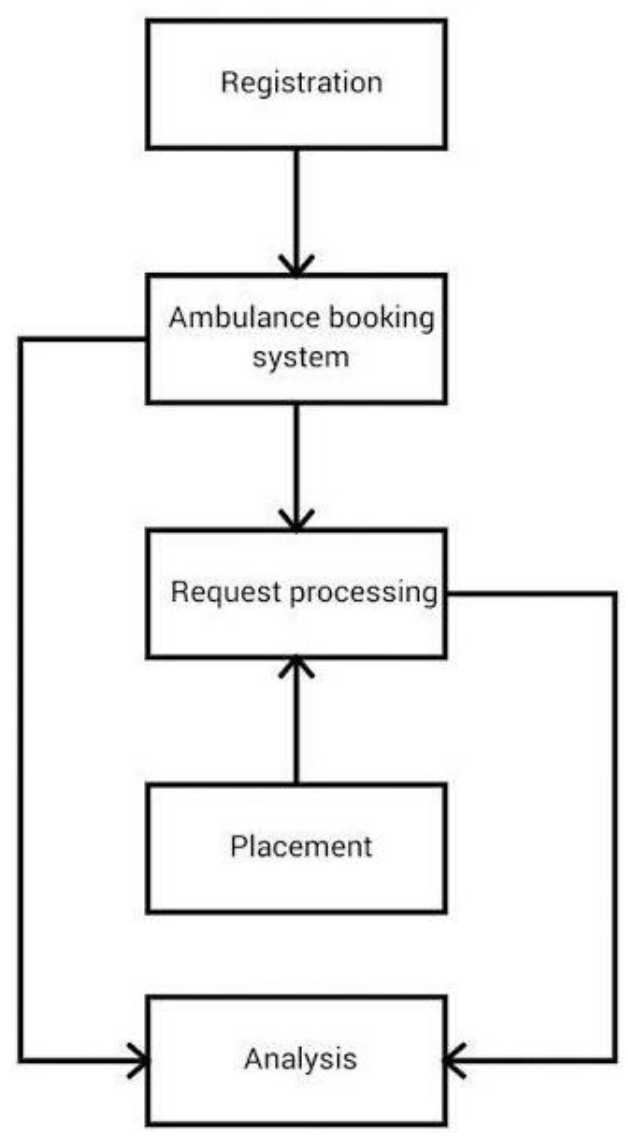

Figure1. Proposed system block diagram

\subsection{Registration}

Registration module is designed for the system users, the hospitals who wants to provide ambulance and the ambulance providers. Anyone who is willing to use this system should register with the system. The pre-registered information of the users reduces the response time, thus proving better service at times of emergency. In addition to the ambulance provided by the ambulance providers, we also manage ambulances of hospitals, which helps to serve maximum people efficiently when there is flooding of requests. Registration for ambulance enable the system to send shortest path to the respective drivers. Thus the Registration module authenticate the users before providing services.

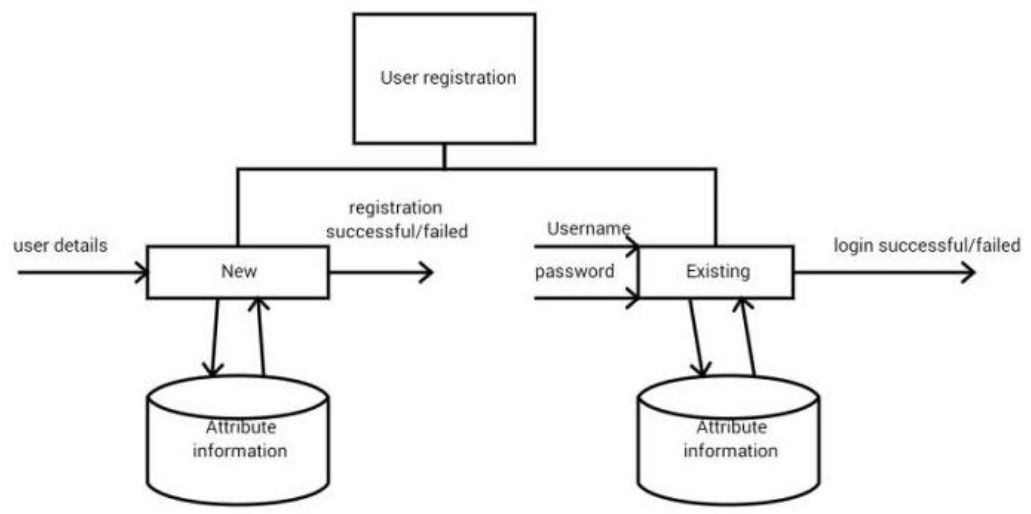

Figure2. User registration modular diagram

\subsection{Ambulance Booking}

This module helps to improve the situation assessment of the operators by relying less on oral communication. The emergency situation is monitored by the pre-registered information and real time location [4]. A client web based application is designed from which user can book the Ambulance. User needs to provide the location by enabling GPS and allowing the system to access their location 
or they can enter the location in the given textbox. The type of ambulance is entered by the user along with severity and number of people affected. After completing all the necessary steps the request is sent to the system. This module uses Google map API [5] to provide the location coordinate by using Geocoding. Users are notified about the allocated ambulance and the estimated time.

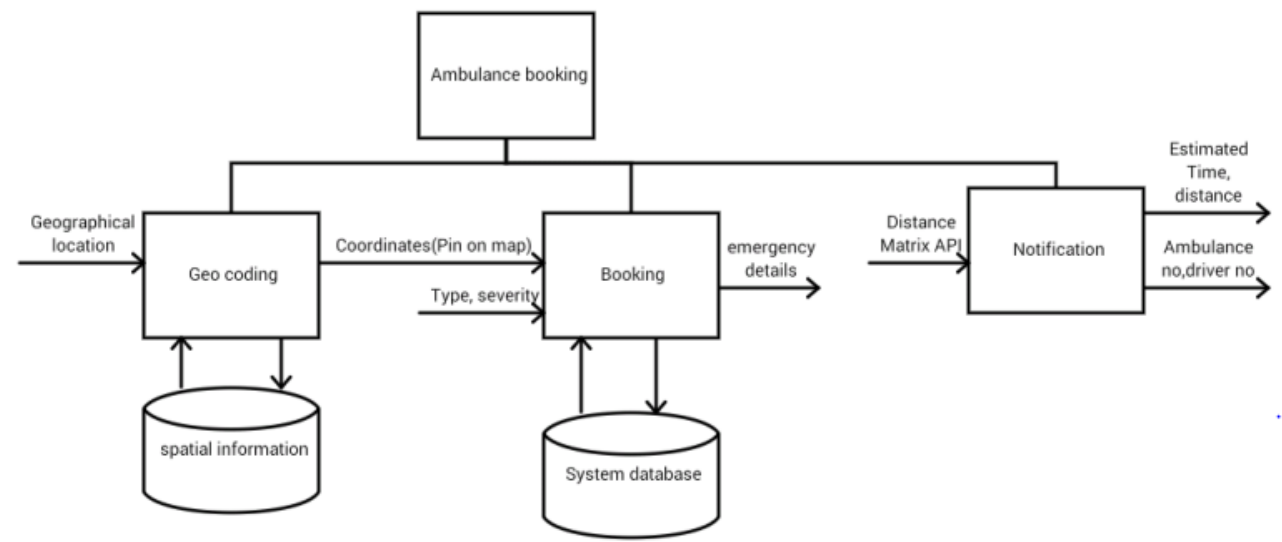

\subsection{Request Processing}

Figure3. Ambulance booking modular diagram

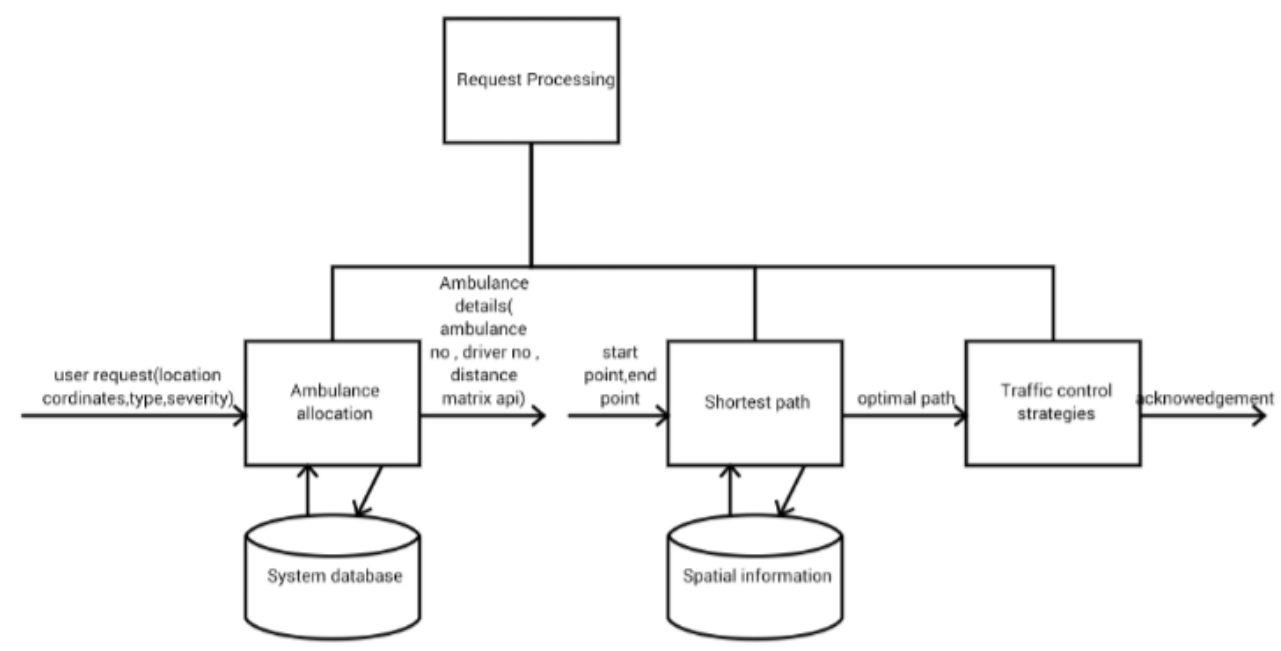

Figure4. Request processing modular diagram

Request processing module processes the request made by the user and allocates the ambulance to it by taking into consideration a lot of factors. The request details contain the location of the user or the where the ambulance is required along with the type of ambulance (cardiac or accident) and the severity of it. The nearest ambulance is allocated to the user. Once the ambulance reaches to the patient, it is provided with the shortest path to the hospital. While providing shortest path, the real time traffic is taken into consideration using google maps api [5]. In case of extreme emergency if the expected time is high due to traffic, then the path details are sent to Traffic Control Room for possible path clearance [6].

\section{- Ambulance Allocation}

Admin can view the location and has the control of all the ambulances. The admin is notified about the status of the ambulance if they are free or not, about the new requests and the hospitals to which the patient is being taken. The papers [3], [7] and [8] compare a number of different strategies from which greedy strategy perfectly fits into proposed system.

\section{Greedy Strategy}

The new request is served by the nearest ambulance from the user location. The current ambulance allocated is replaced with the one from the base if available [9]. Once the patient is in the ambulance, it is sent to the nearest hospital, which is selected based on the type of emergency. After completing the ride from patient to hospital the ambulance is sent to the nearest base for sanitization if needed. Greedy algorithm from [10] is used for ambulance allocation. 


\section{- Shortest Path}

The shortest path to the ambulance driver is provided using google map's distance matrix api[5]. Shortest path is provided from site of ambulance to the site where ambulance is called and from that site to the hospital. This shortest path is calculated with respect to time and given to the TCR.

\section{- Traffic Control Strategy}

The strategy proposed in [11] and [12], to turn signal green which would further make situation go worse if two ambulance approach from different directions, hence it is not feasible in a country with second highest population In order to deal with the rising traffic on roads, the system sends the shortest path calculated for the ambulance to the Traffic Control Room. The system in TCR displays the route along with real time traffic data. TCR controller monitors the route for traffic jams and tries to clear the jams by contacting the respected personnel [6]. TCR returns whether it can clear the path or not.

IF (TCR clears the route)

returns YES

ELSE

returns NO

\subsection{Initial Placement of Ambulance}

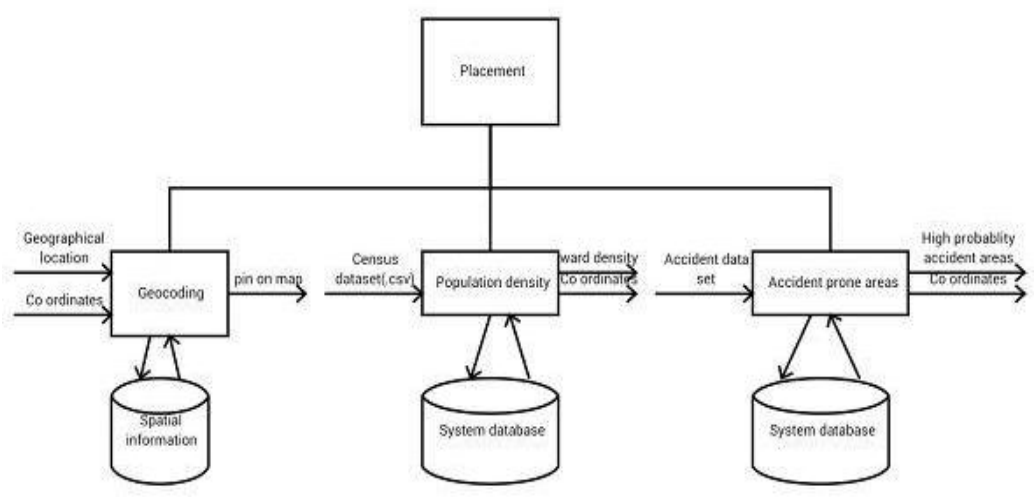

Figure5. Placement module diagram

Current location of ambulances affects its availability during emergencies. The proposed model for initial ambulance placement has the aim to cover all the points such that each site covered within the travel distance from the preset time with a minimum number of ambulances.

The initial placement of cardiac ambulances is based on population density data [13] and [14] the number of users of the system. The area which has higher middle aged and old aged population has higher chances than the area which is sparse in population.

The initial placement of ambulances for accident patients is based on accident prone area data [15]. There will be at least one ambulance within $2 \mathrm{~km}$ radius of the accident prone area.

\subsection{Analysis}

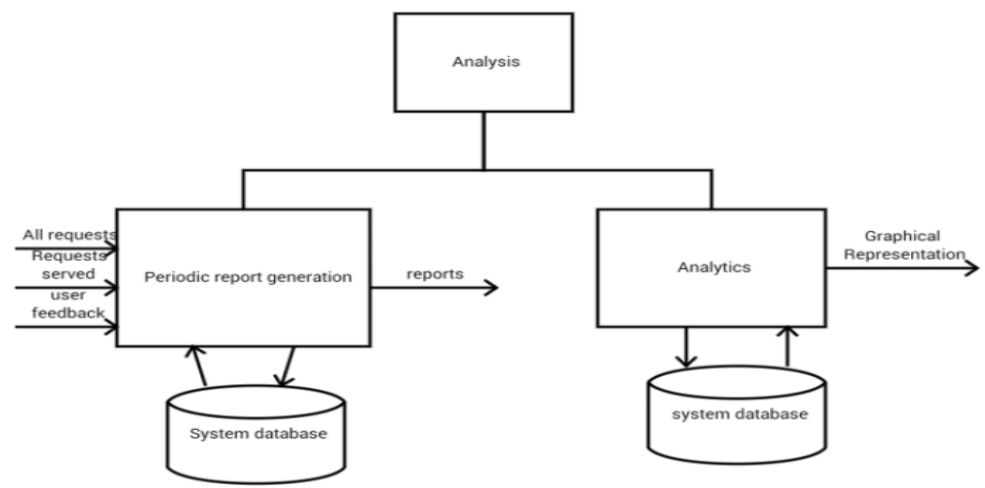

Figure6. Analysis modular diagram 
This module is responsible for testing the efficiency of the system. Monthly/periodic reports will be generated. These reports will consider the no. of requests received, not of shortest path provided, no. of requests processed, No. Of lives saved, total cost, no. of requests to TCR and no. of times, routes cleared by TCR, overall ratings and review by customers [16]. Pie charts, graphs will be generated monthly.

Let the total no. of requests be $\mathrm{N}$ and the no. of requests processed be $\mathrm{P}$, then

Request Acceptance Ratio (Ar) $=\mathrm{P} / \mathrm{N}$

The Request Acceptance Ratio (Ar) will be between 0 and 1. For higher efficiency it should be as close as possible to 1 .

Let the no. of shortest path allocated be S, and no. of times ambulances reached in expected time be T, then

Efficiency of the system $=\left(\mathrm{P}^{*} \mathrm{~T} * 100\right) /(\mathrm{S} * \mathrm{~N})$

Efficiency will be in percentage. If the efficiency of the system in initial month is higher than $85 \%$, then it can create a possible difference using technology for social good

\section{CONCLUSION}

Various challenges faced by the existing ambulance system and the difficulties faced by them in day to day life are considered in this paper. Based on that a new model is proposed in order to reduce to the reaction time of processing request and increasing the accuracy of the system by providing a software for emergency system. The system makes use of Google map Api for shortest path, provides better allocation strategies and coordinate with Traffic control room in cases of extreme traffic. Thus, it allows the system to serve maximum users.

\section{REFERENCES}

[1] Dah-Ming Shiah and Shu-Wen Chen“Ambulance Allocation Capacity Model”,2007

[2] P. Arunmozhi, P. Joseph William "Automatic Ambulance Rescue System Using Shortest Path Finding Algorithm", Volume 3 Issue 5,2014

[3] Niklas Meinzer and Sabine Storandt "Decision Support in Emergency Medical Systems: New Strategies for Dynamic Ambulance Allocation" Albert-Ludwigs-University,2014

[4] Odd Erik Gundersen, Fredrik Øvergaard, Jannicke Røren.’Towards Reducing the Reaction Time of Emergency Services through Improved Situation Assessment”,2016

[5] Google Maps Website https://developers.google.com/maps/

[6] S. Djahel, M. Salehie, I. Tal, and P. Jamshid. "Adaptive Traffic Management for Secure and Efficient Emergency Services in Smart Cities". IEEE PerCom (WiP track), San Diego, California, USA, March 18-22, 2013

[7] Yisong Yue and Lavanya Marla and Ramayya Krishnan, "An Efficient Simulation-Based Approach to Ambulance Fleet Allocation and Dynamic Redeployment “

[8] Restrepo, M.; Henderson, S. G.; and Topaloglu, H. 2009 Erlang loss models for the static deployment of ambulances. Health care management science 12(1):67-79

[9] Pillac, V.; Gendreau, M.; Guret, C.; and Medaglia, A. L. 2013. A review of dynamic vehicle routing problems. European Journal of Operational Research 225(1):1 - 11

[10] Yisong Yue and Lavanya Marla and Krishnan, Ramayya Krishnan," An Efficient SimulationBased Approach to Ambulance Fleet Allocation and Dynamic Redeployment “, 2012

[11] Soufiene Djahel, Nicolas Smith, Shen Wang, and John Murphy," Reducing Emergency Services Response Time in Smart Cities: An Advanced Adaptive and Fuzzy Approach", 2015.

[12] Tandrima Chowdhury Smriti Singh S. Maflin Shaby "A Rescue System of an advanced ambulance using prioritized traffic switching”, Sathyabama University, 2015

[13] https://github.com/mickeykedia/Mumbai-Population-Map/blob/master/README.md

[14] http://censusindia.gov.in/pca/pcadata/Houselisting-housing-Maha.html

[15] Sachin Kumar and Durga Toshniwal," A data mining framework to analyze road accident data", 2015

[16] B.Ganeshkumar, D.Ramesh "Emergency Response Management and Information System (ERMIS) - A GIS based software to resolve the emergency recovery challenges in Madurai city", 2010 